\title{
Dual-delivery of vancomycin and icariin from an injectable calcium phosphate cement-release system for controlling infection and improving bone healing
}

\author{
JIAN-GUO HUANG, LONG PANG, ZHI-RONG CHEN and XI-PENG TAN \\ Orthopaedics Department Ward 3, General Hospital of Ningxia Medical University, \\ Yinchuan, Xingqing 750004, P.R. China
}

Received March 8, 2013; Accepted July 23, 2013

DOI: $10.3892 / \mathrm{mmr} .2013 .1624$

\begin{abstract}
Infectious bone diseases following severely contaminated open fractures are frequently encountered in clinical practice. It is difficult to successfully repair bone and control infection at the same time. To identify a better treatment method, we prepared a dual-drug release system that was comprised of icariin (IC, a natural osteoinductive molecule), vancomycin (VA) and injectable calcium phosphate cement (CPC). The ultrastructure of the dual-drug release system was evaluated by scanning electron microscopy and the biocompatibility was assessed by cell culture. In addition, the release kinetics of IC and VA were respectively investigated by using high-performance liquid chromatography. Finally, this system was used to repair Staphylococcus aureuscontaminated bone defects in a rabbit model. Twelve weeks after the implantation of IC-VA/CPC, the contaminated bone defects were completely repaired, with significantly improved formation of lamellar bone and recanalization of the marrow cavity compared with the controls (CPC without antibiotics or osteoinductive agent). These results demonstrate that this dual-drug release system, with its concomitant antibiotic and osteoinductive properties, has significant potential for the treatment of contaminated bone injury or infectious bone disease.
\end{abstract}

\section{Introduction}

Open fractures, particularly those that are severely contaminated, frequently result in infection-related bone diseases, such as osteomyelitis, nonunion and infectious bone defects. It is difficult to effectively repair bone and control infection

Correspondence to: Dr Jian-Guo Huang, Orthopaedics Department Ward 3, General Hospital of Ningxia Medical University, 804 Shengli South Street, Xingqing, Yinchuan, Ningxia Hui Autonomous Region 750004, P.R. China

E-mail: huangjg75@163.com

Key words: controlled release, icariin, vancomycin, calcium phosphate cement, infection at the same time. As infection is favored by the devitalization of bone, soft tissue and loss of skeletal stability, systemic administration of antibiotics is unable to achieve a sufficient local drug concentration. However, long-term administration of antibiotics may give rise to side-effects, including myelosuppression, nephrotoxicity and drug-induced hepatitis (1). Conventional treatments, such as surgical debridement and suction irrigation, are only able to control local infection. For patients with nonunion or bone defects, these treatments require two stages; control of infection followed by bone grafting (2-4). Therefore, the high cost and length of treatment cause suffering of the patients. Another conventional clinical procedure for treating severely contaminated open fractures is the application of antibiotic-loaded poly(methyl methacrylate) (PMMA) cement beads, which are generally used as a local antibiotic release system (LARS) and have been shown to decrease infection in a number of clinical studies (5). However, PMMA is non-biodegradable and cannot be allowed to remain in the wound bed during definitive closure, thus it requires removal during a second surgical step. At the time of definitive closure, any remaining defect or fracture is repaired by using other methods (6).

To overcome the limitations of non-biodegradable PMMA and the other two-stage treatment methods, several biodegradable materials, whether they are solid or injectable, have been widely investigated as scaffolds for the release of various drugs in LARS $(1,6)$. In contrast to solid and prefabricated scaffolds, injectable scaffolds hold great promise for the treatment of infectious bone disease. Firstly, injectable scaffolds may take the shape of the irregular cavities or bone defects after routine debridement. Furthermore, antibiotics or growth factors may be easily incorporated into the solution by mixing prior to the injection. Calcium phosphate cement (CPC) is one type of injectable scaffold, which is obtained by mixing tricalcium phosphate or so-called amorphous calcium phosphate powders with an aqueous solution to form a paste that hardens within a restricted period of time (15-30 min) under low-processing temperatures $(7,8)$. Due to its good osteoconductivity, CPC has been used as a scaffold of LARS for the controlled release of antibiotics and has been shown to release more antibiotics over a longer period compared with PMMA (9). Furthermore, for the treatment of complicated fractures, bone defects and 
nonunion, the improvement of osteoinductive properties is also required in these scaffolds.

To improve the bone repair capability of scaffolds (e.g., CPC), osteoinductive agents, such as growth factors (GFs) and biological molecules, are usually administered in the LARS. However, the high cost and rapid degradation of such expensive GFs limit their widespread use, particularly in clinics (10). Therefore, there is an urgent need to develop alternative osteogenic products or drugs with higher efficacies and lower costs than GFs (11). Certain herbal medicines have been widely used in the treatment of fractures and bone disorders for thousands of years in Asia $(12,13)$. Herba epimedii is one of the most frequently used herbs in formulas that are prescribed for the treatment of osteoporosis in China, Japan and Korea (13). Icariin (IC; molecular formula, $\mathrm{C}_{33} \mathrm{H}_{40} \mathrm{O}_{15}$; molecular weight, $676.67 \mathrm{~g} / \mathrm{mol}$ ) is a flavonoid isolated from Herba epimedii and is considered to be the major (77\%) bioactive component of this herb (14). IC was shown to have markedly positive effects on the proliferation of osteoblasts in numerous studies $(12,14)$. It also showed a marked effect on the ossific differentiation of bone marrow-derived stromal cells (BMSCs), which was demonstrated to occur in a BMP and core binding factor $\alpha-1$ (Cbfa-1)-dependent manner (15).

In the present study, we developed a dual-drug release system that comprised of IC, vancomycin (VA) and CPC. In the evaluation of this new system, we hypothesized that IC-VA/CPC, with its controlled antibiotic release kinetics and osteoinductive capabilities, may concomitantly control infection and improve bone healing when applied in infected bone segmental defects. To test this hypothesis, we examined the ultrastructure, biocompatibility and drug release profiles of IC-VA/CPC. Subsequently, this system was implanted into an infected segmental radial defect in a rabbit model. We specifically evaluated the ability of IC-VA/CPC to treat infected bone defects.

\section{Materials and methods}

Preparation of IC-VA/CPC system. The system was prepared as described previously (16). Briefly, IC (National Institute for the Control of Pharmaceuticals and Biological Products, Beijing, China) was dissolved into ethanol to prepare an IC solution at a concentration of $8 \mathrm{mg} / \mathrm{ml}$. A solution of vancomycin hydrochloride (VA; molecular formula, $\mathrm{C}_{66} \mathrm{H}_{75} \mathrm{C}_{12} \mathrm{~N}_{9} \mathrm{O}_{24} \cdot \mathrm{HCl}$; molecular weight, $1485.71 \mathrm{~g} / \mathrm{mol}$; Sigma, Shanghai, China) was also prepared by dissolving VA in phosphate-buffered solution (PBS, $\mathrm{pH} 8.0$ ) at a concentration of $80 \mathrm{mg} / \mathrm{ml}$. The two solutions were then mixed at a ratio of $1: 1(\mathrm{v} / \mathrm{v})$. The IC-VA mixture was then stirred for $2 \mathrm{~h}$, followed by the slow addition of CPC powder (Shanghai Rebone Biomaterials Co., Ltd., Shanghai, China) to prepare an IC-VA/CPC paste precursor at a liquid/powder ratio of 1:1 (ml/g). Subsequently, the precursor was homogenized by $4 \mathrm{~h}$ vigorous stirring. The resulting solution was cast by a glass mold $(4 \mathrm{~mm}$ in diameter, $15 \mathrm{~mm}$ in length) and placed at $4^{\circ} \mathrm{C}$ for $6 \mathrm{~h},-10^{\circ} \mathrm{C}$ for $3 \mathrm{~h}$, then freeze-dried to obtain IC-VA/CPC cylinders. Each cylinder contained $\sim 2 \mathrm{mg}$ of IC and $20 \mathrm{mg}$ of VA. We also prepared a cylinder of IC/CPC or VA/CPC at the same concentration of VA or IC for the animal experiments. A pure CPC cylinder was used as a control in the animal experiment and biocom- patibility tests. All cylinders were sterilized with $20 \mathrm{kGy}{ }^{60} \mathrm{Co}$ and stored in vacuum packages at room temperature prior to subsequent use.

Ultrastructure examination. The microstructure of the cylinder samples was examined using a scanning electron microscope (SEM, S-3000N; Hitachi, Japan) after the samples were sputter-coated with gold under a vacuum.

Biocompatibility analysis. Biocompatibility was determined by evaluating the toxicity of sample extracts (according to ISO10993-5) and the survival of cells seeded directly onto the sample surfaces (17). Briefly, sample extracts of IC-VA/CPC and CPC were created in Dulbecco's modified Eagle's medium (DMEM; Sigma) without fetal calf serum (FCS) at a concentration of $100 \mathrm{mg} / \mathrm{ml}$ (extract/DMEM culture medium) for $24 \mathrm{~h}$ at $37^{\circ} \mathrm{C}$ in a $5 \% \mathrm{CO}_{2}$ atmosphere, following the guidelines of ISO standard 10993-12. Balb/c 3T3 cells (American Type Culture Collection, Manassas, VA, USA) were cultured in DMEM containing ampicillin $(0.025 \mathrm{~g} / \mathrm{l})$ and streptomycin $(0.1 \mathrm{~g} / \mathrm{l})$ supplemented with $10 \% \mathrm{FCS}$ (Sigma) at $37^{\circ} \mathrm{C}$ in a $5 \% \mathrm{CO}_{2}$ atmosphere. The tests were performed using 24-well plates, by plating $3 \mathrm{~T} 3$ cells at a density of $3 \times 10^{4}$ cells/well. Cells plated at the same density in wells without any extracts were used as blank controls. The total cell number of the four groups at days 1, 3, 5 and 7 was estimated by quantifying the double-stranded DNA (dsDNA) content of each sample using a PicoGreen dsDNA Quantification kit (Molecular Probes, Eugene, OR, USA). The total dsDNA was extracted by enzymatic digestion and assayed according to the manufacturer's instructions. The proliferation of the cells was interpreted by changes in dsDNA quantity. In order to observe the morphology of cells cultivated on the surface of granules, 3T3 cells were plated onto the samples at a density of $5 \times 10^{4}$ cells/sample. At 24 and $72 \mathrm{~h}$ after plating, the cells were fixed with modified Karnovsky's solution and post-fixed with $1 \%$ osmium tetroxide in $0.05 \mathrm{M}$ cacodylate buffer $(\mathrm{pH} 7.2)$ for $1 \mathrm{~h}$ at room temperature. The samples were washed with distilled water, dehydrated using increasing solutions of ethanol and critical point drying, metallized with gold and analyzed under the SEM.

Release kinetics of IC or VA from IC-VA/CPC. The release behaviors of IC and VA from the IC-VA/CPC system were measured by high-performance liquid chromatography (HPLC) with UV detection at $230 \mathrm{~nm}$ (Agilent1200; Agilent Technologies, Santa Clara, CA, USA) according a previous method with modifications (18). Briefly, samples

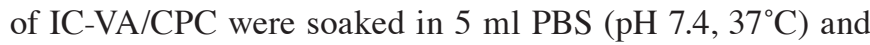
agitated gently at $10 \mathrm{rpm}$. At $0,1,3,5,7,10,15,20,25$ and 30 days, $5 \mathrm{ml}$ PBS was collected (stored at $4^{\circ} \mathrm{C}$ for HPLC examination) and replaced by adding the same amount of fresh PBS. For analysis of IC concentration, the samples were centrifuged $(1,000 \mathrm{x} \mathrm{g})$ for $10 \mathrm{~min}$ and $0.5 \mathrm{ml}$ supernatants were isolated. After the addition of $0.5 \mathrm{ml}$ acetonitrile, the mixed solution was re-centrifuged $(4,000 \mathrm{x} \mathrm{g})$ for $10 \mathrm{~min}$ and $20 \mu \mathrm{l}$ supernatants were applied for HPLC analysis. Peak area data was calculated by integration using Empower 2 software (Waters Co., Milford, MA, USA), which identifies the absorbance peaks of different proteins and calculates the area of the 

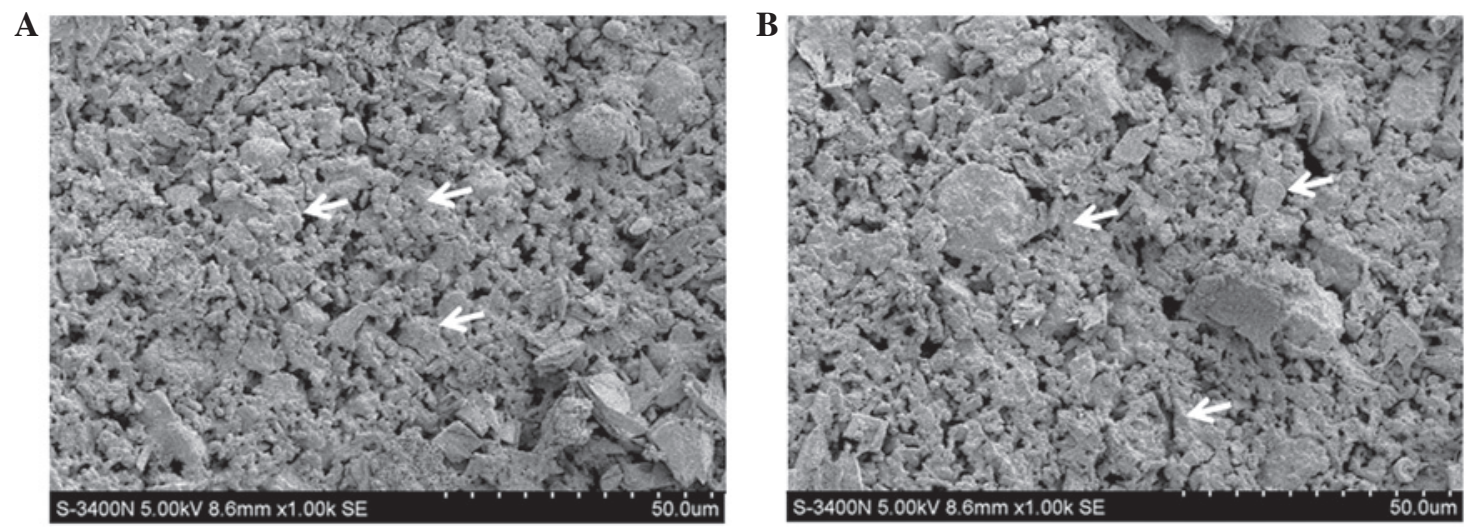

Figure 1. (A) SEM showed the granules (arrow) on the surface of calcium phosphate cement (CPC). (B) IC-VA/CPC were ball-like, and spongy pores formed among the granules. SEM, scanning electron microscope; IC, icariin; VA, vancomycin.

peak, therefore determining the concentration of the proteins. IC or VA released from the sample was calculated according to the standard IC level of VA solutions and the percentage of IC or VA released from IC-VA/CPC was assessed. Each test was replicated three times $(n=5)$.

IC-VA/CPC repairs osseous defects in a rabbit model. Forty-eight male New Zealand white rabbits weighing 2.2-2.6 kg (male and 6 months old) were randomly divided into 4 groups, and each group contained 12 animals. Under general anesthesia (i.v. injection of $15 \mathrm{mg} / \mathrm{kg}$ pentobarbital) an osteoperiosteal defect of $15 \mathrm{~mm}$ was created through the whole thickness of the shaft of the right radius by removing the segmental bone. Staphylococcus aureus (ATCC28923) suspension $(0.2 \mathrm{ml})$ at a concentration of $5 \times 10^{6}$ colony forming units $(\mathrm{CFU}) / \mathrm{ml}$ was injected into each defect. After $60 \mathrm{~min}$, the defects were washed with sterile saline. In group A, the defects were filled with one IC-VA/CPC containing $2 \mathrm{mg}$ of $\mathrm{IC}$ and $20 \mathrm{mg}$ of VA. In groups $\mathrm{B}$ and $\mathrm{C}$, the defects were filled with one IC/CPC (containing $2 \mathrm{mg}$ of IC) and VA/CPC (containing $20 \mathrm{mg}$ of VA), respectively. The defects of group D were left empty without any filling material as a blank control. The muscles held the graft in place without the need for internal fixation or external splints. At 4, 8 and 12 weeks, four animals from each group were sacrificed for radiological and histological evaluation. All animal experiments were conducted according to the Chinese Regulations of Animal Welfare and permission was granted by the Ethics Committee of the Ningxia Medical University

\section{Results}

Ultrastructure examination. The samples of CPC (Fig. 1A) and IC-VA/CPC (Fig. 1B) both exhibited three-dimensional structures with numerous granules. The SEM images demonstrated that the loading of IC and VA caused no clear changes on the ultrastructure of CPC. Using SEM, it was also observed that the granules contained in the samples were $\sim 5-10 \mu \mathrm{m}$ and ball-like or irregular, and were formed on the surface and in the middle of the samples. Furthermore, among the granules, numerous spongy pores were formed, which is hypothesized to be helpful for the degradation of the scaffold as well as the release of drugs.
Biocompatibility analysis. The proliferation of the cells, which were co-cultured with CPC and IC-VA/CPC, was interpreted by changes in dsDNA quantity. In the CPC and blank control groups, cells proliferated faster during the first 3 days and plateaued at day 5 , as indicated by the change in dsDNA content. By contrast, the dsDNA contents of IC-VA/CPC increased rapidly from 1 to 7 days. Between days 3 and 7 , the dsDNA contents of the IC-VA/CPC group were significantly higher than those of the CPC and blank control groups $(\mathrm{P}<0.05)$. There were no significant differences in dsDNA content between the CPC and blank groups ( $\mathrm{P}>0.05$; Fig. 2A). These findings were further corroborated by SEM analysis. One day after the seeding of 3T3 cells on the CPC and IC-VA/CPC, cells appeared flattened, polygonal and spindle-shaped and were distributed evenly. At 7 days, the number of cells on the surface of IC-VA/CPC (Fig. 2B) was increased compared with that on the surface of CPC (Fig. 2C). A number of cells on the IC-VA/CPC had spread and colonized patches of the CPC surface. The spread cells maintained physical contact with each other through filopodia or lamellipodia.

Release kinetics of IC and VA from IC-VA/CPC. The drug release behavior of the IC-VA/CPC system in vitro was investigated by HPLC examination and then calculated based on a standard curve, and was demonstrated as the accumulated percentage of IC and VA release, respectively (Fig. 3). The IC-release profile exhibited a low burst of rapid drug release. From 0 to 7 days, $235 \%$ IC was released and then the speed decreased and $<50 \%$ of IC was released by 25 days. From 25 days, there was a second rapid release and 30 days later there was $\sim 15 \%$ of IC remaining in the system (Fig. 3A). The VA-release profile showed a modest burst of rapid drug release. From 0 to 5 days, $\sim 45 \%$ VA was released and then the speed decreased and $\sim 90 \%$ VA was released by 30 days (Fig. 3A).

Osseous defect repaired by IC-VA/CPC. All wounds in the rabbits of group A (IC-VA/CPC) and group C (VA/CPC) healed completely within 10 days. The wounds of group D (blank control) showed delayed healing of 3-5 weeks, while the wounds in group B (IC/CPC) failed to heal after $>5$ weeks. The wounds of certain rabbits in groups B and D appeared red and swollen for $>7$ days, and there was pus exuding from the wounds. Upon bacteriological examination, S. aureus 
A

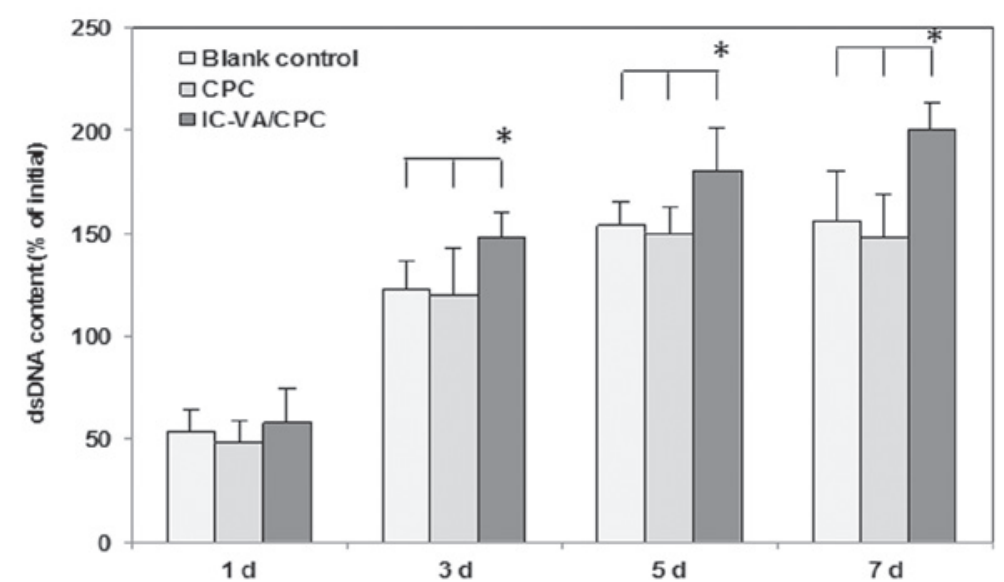

B

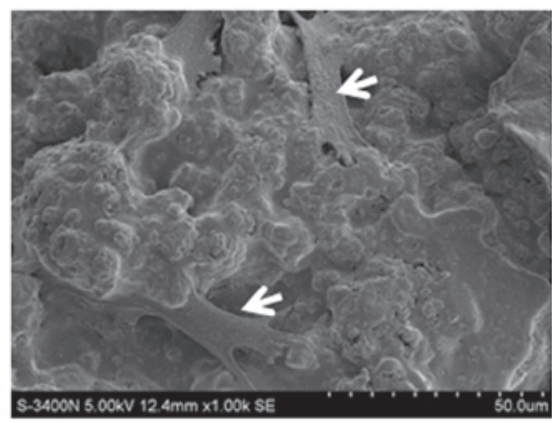

C

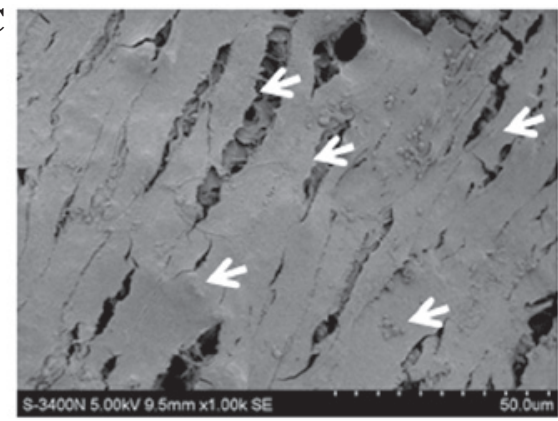

Figure 2. (A) Double-stranded (ds)DNA content of cells in the sample extracts of calcium phosphate cement (CPC) and IC-VA/CPC. " $\mathrm{P}<0.05$ ( $\mathrm{n}=15)$. (B) Scanning electron microscope (SEM) image of cells on the surface of IC-VA/CPC (arrow, cells). (C) SEM image of cells on the surface of CPC (arrow, cells). IC, icariin; VA, vancomycin.

A

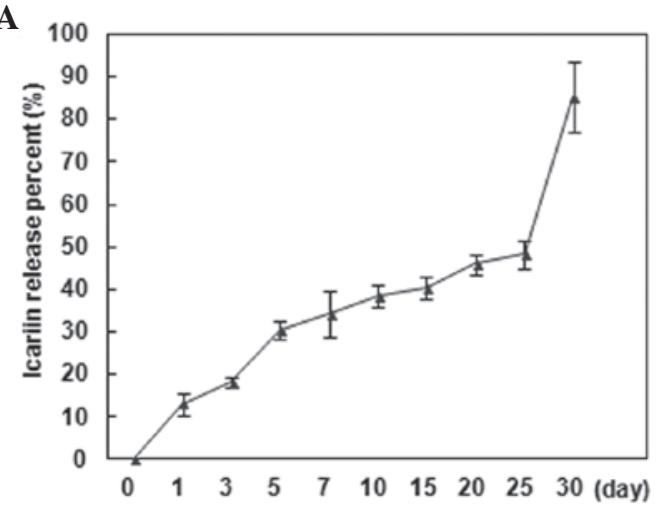

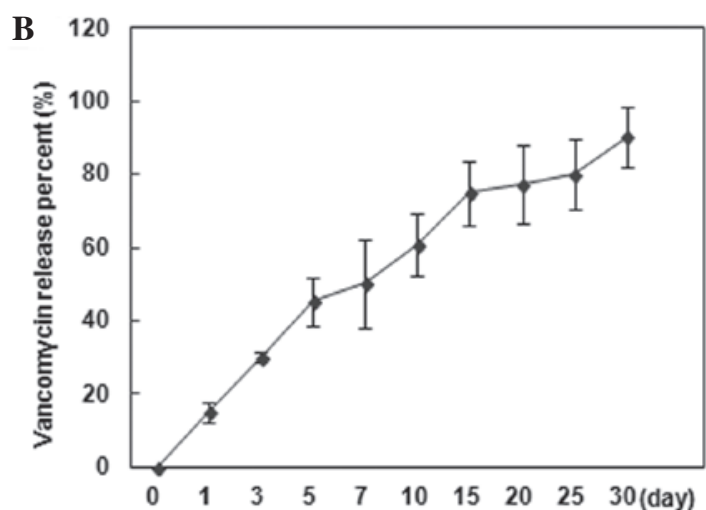

Figure 3. Release behavior of (A) icariin (IC) and (B) vancomycin (VA) from the IC-VA/CPC system in vitro. CPC, calcium phosphate cement.

ATCC28923 was detected in the secreted pus. Pus secretion in the wound area stopped and was absorbed without any treatment in $>3$ weeks. Of the total 48 rabbits used in this study, three animals died; on the 50th day (one animal in group D), the 10th day (one animal of group B) and the 14th day (one animal of group B), respectively. Blood samples from the dead rabbits, collected from the heart under sterile conditions, were cultured for $48 \mathrm{~h}$ and $S$. aureus was detected. Twelve weeks after surgery, $S$. aureus could still be detected in the bone defects of rabbits from groups B (9/10) and D (2/11) by tissue culture, but not in any animals of groups $\mathrm{A}$ and $\mathrm{C}$.

Through radiological observation, a standard defect shadow was observed at the radius of rabbits in group $\mathrm{D}$, while in groups $\mathrm{A}, \mathrm{B}$ and $\mathrm{C}$, the defects were filled with high-density material, as revealed by the postoperative radiograph. In animals of group A, a bone callus formed by the 4th week, defects started repairing by the 8 th week and the medullary cavity was recanalized by the 12th week. By comparison, the defects appeared to be delayed union or nonunion in group $\mathrm{C}$ (delayed union, 7; nonunion, 5) and group D (nonunion, 11), and osteomyelitis was observed in group B (10/10) at week 12 (Fig. 4).

In accordance with the radiological examination, different changes were observed in the four groups by histological evaluation. In group A, chondrogenesis and osteogenesis were observed, while no indications of inflammatory infiltration were found 4 weeks postoperatively. On the 8th week, copious new bone tissue was observed within and around the material. There was clear creeping substitution in some areas and the 


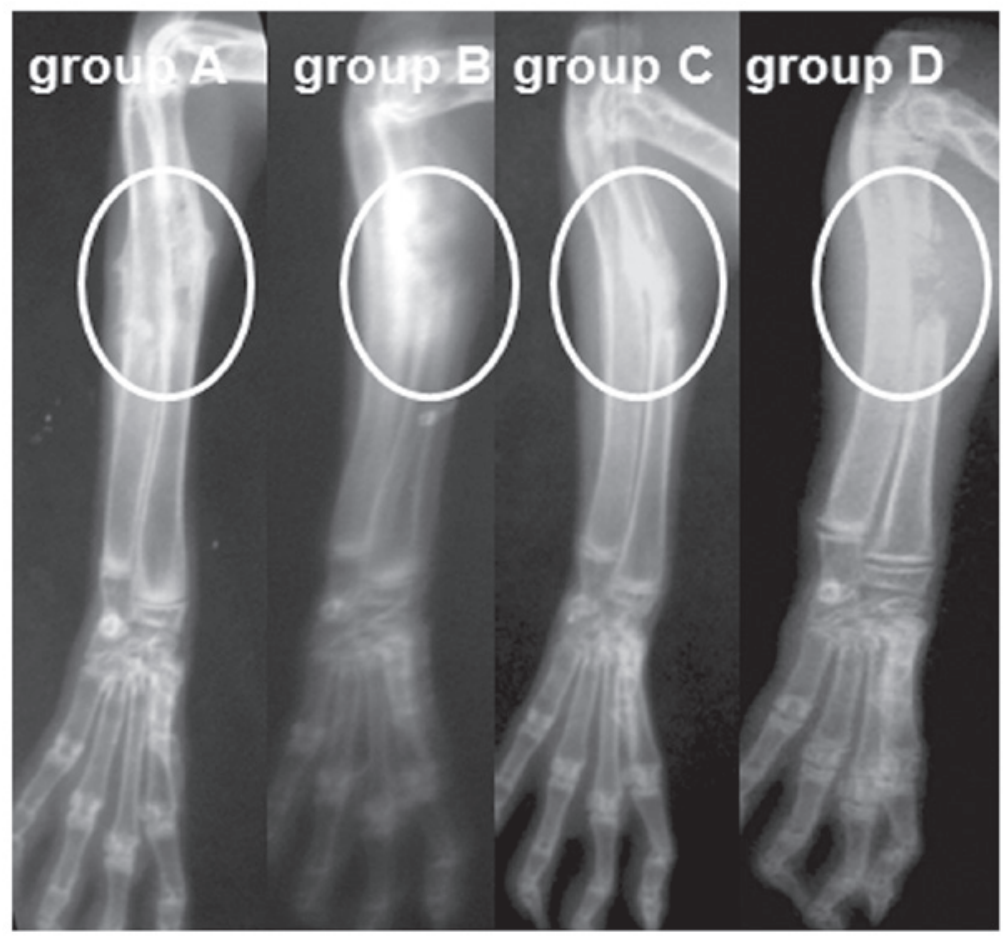

Figure 4. At the 12th week, bone defects were repaired and the medullary cavity was recanalized in group A. In comparison, osteomyelitis formed in group B, nonunion of bone occurred in group $\mathrm{C}$ and no bone formed in the defect of group $\mathrm{D}$ (circle, defect area).

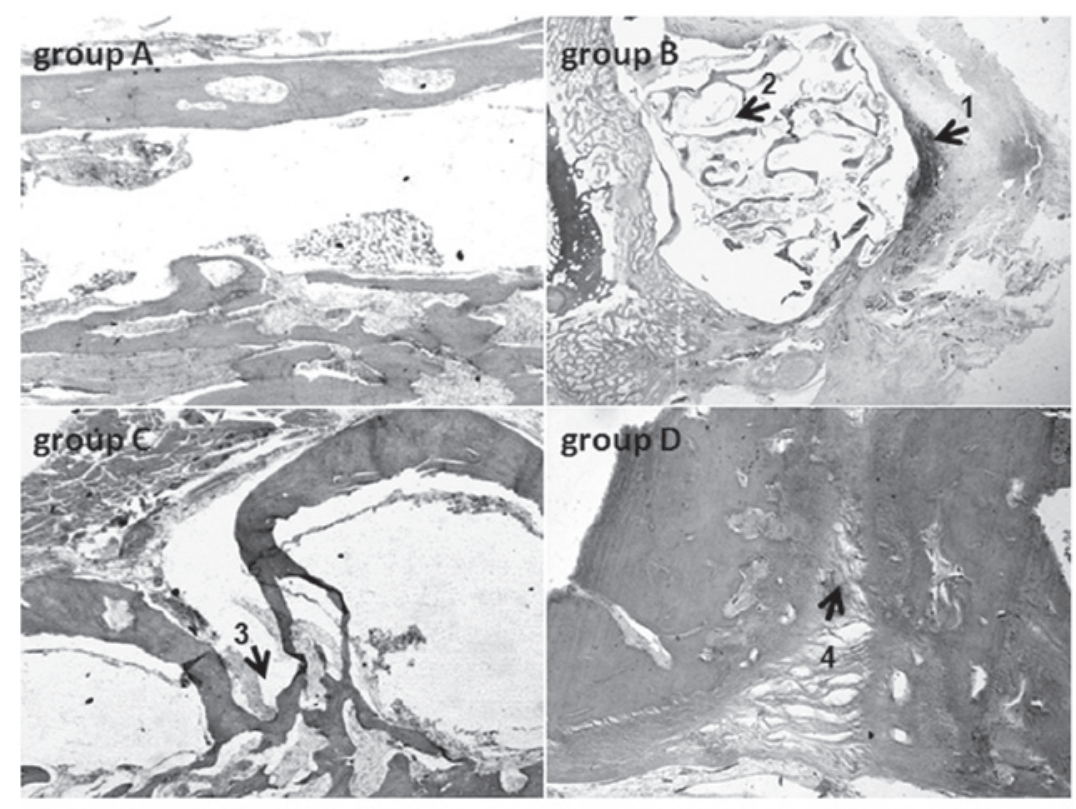

Figure 5. At the 12th week, ossification occurred and the medullary cavity recanalized in group A. In group B, infiltration of chronic inflammatory cells (arrow 1, inflammatory cells) and numerous incompletely resorbed and degraded remnants (arrow 2, undegraded materials) were noted. Nonunion was found in group C (arrow 3, nonunion area) and bone defects were filled with soft tissue in group D (arrow 4, scar tissue). Magnification, x100.

graft was disorganized, largely resorbed and combined with the new bone. The woven bone, lamellar bone and recanalization of the marrow cavity were identified in the majority of the specimens, and the defects were found to have been completely repaired by the 12th week. In group B, animals showed signs of infiltration of chronic inflammatory cells, while the remaining material degraded into small fragments and was embedded in the connective tissue. In group C, a number of animals (7/12) had bone defects that were filled with scar tissue, while the other animals (5/12) had repaired defects. In group D, the bone defects of the animals (11/11) were filled with scar tissue (Fig. 5).

\section{Discussion}

In the present study, we firstly prepared the solution of IC and VA, respectively, at various concentrations and then mixed 
them with PCP powder to create a precursor paste. Subsequent homogeneous distribution of the drugs was obtained by stirring, and spongy pores were also formed at that time. After freeze-drying, the two drugs were completely absorbed by $\mathrm{CPC}$, which had the lowest effect on the porosity of the composite. Moreover, in situ composition CPC paste may contain homogeneous dispersed granules, which are easily aggregated and hard scattered. Using water as a pore-forming agent, the freeze-drying technique contributed greatly to the production of satisfactory micro-pores (11).

The requirements of injectable scaffolds are numerous, but the most important factor is that they must be biocompatible. CPC has been shown to have good biocompatibility and possess excellent osteoconductivity and bone replacement capability. Due to its highly promising potential for use in a number of restorative dental and craniofacial procedures, CPC was approved by the Food and Drug Administration (FDA) for craniofacial indications a decade ago (19). IC has been shown to have a number of positive effects on the proliferation of osteoblasts and BMSCs in several studies (14-15). In the present study, we used 3T3 cells for the biocompatibility test according to the ISO standard (10993-12) (20) and found that IC did not have any negative effects on the biocompatibility of CPC. Notably, the introduction of IC to CPC appeared to have much improved effects on the proliferation of 3T3 cells; the number of surviving cells cultured in the IC-VA/CPC extracts was higher than that cultured in the pure CPC extracts and blank control at the seventh day of culture. We hypothesize that the mechanism may be related to the mitogen-activated protein kinase (MAPK) signaling pathways (21), and further, more detailed studies are required. VA has been reported to have a less negative effect on new bone formation than other antibiotics and has also been demonstrated not to compromise the healing effect of BMP-2 in a non-infected segmental defect model (6). Based on the results of the biocompatibility test, we hypothesize that VA at the concentration used in this study also has no negative effect on the proliferation of $3 \mathrm{~T} 3$ cells.

For the regeneration of bone tissue, an ideal local antibiotic release system should serve two primary roles; as a delivery carrier to provide maximal antibiotics for infection control without causing side-effects, such as myelosuppression, nephrotoxicity and drug-induced hepatitis, and as an osteoconductive scaffold with suitable pore structure for osteoinductive agent loading and bone formation (16). The optimal release kinetics of the high dose of osteoinductive agents and the co-delivery of antibiotics should overcome the detrimental effects of bacteria on bone healing. In the present study, we observed that CPC allowed the dual-delivery of VA and IC in the local area of injury for more than 30 days, which is hypothesized to be helpful for the control of infection and improvement of bone healing at the same time. In particular, the burst release of VA during the first five days should contribute greatly to the inhibition of bacteria.

It is well-known that Staphylococcus strains cause the majority of infectious complications in orthopedics and traumatology (22). Methicillin-resistant S. aureus (MRSA) strains and coagulase-negative staphylococci (MRCoNS) impose a serious limitation on the selection of effective antibiotics in both prophylaxis and therapy. Glycopeptide antibiotics, such as vancomycin and teicoplanin, still maintain their high efficacy against Staphylococcus strains. Resistance to this group of antibiotics remains rare and has only been observed in strains with lower sensitivity to VA (VA intermediate $S$. aureus) and a minimum inhibitory concentration (MIC) of 4-8 mg/l $(20,23)$. In this study, VA was loaded in CPC for the control of infection and was shown to be effective for the treatment of $S$. aureus-infected bone defects (VA/CPC and IC-VA/CPC groups). In contrast to the groups with VA, the groups without VA (IC/CPC and blank control groups) exhibited serious signs of chronic inflammation. Although this model, with an inoculum of $5 \times 10^{6}$ of this strain of S. aureus and $1 \mathrm{~h}$ treatment delay, is not fully susceptible to local antibiotics, it has previously been shown to yield significant levels of bacterial infections (1). Moreover, all animal models are contrived and it is difficult to mimic the clinical scenario. We chose to use an extremely challenging model to ensure that a substantial bio-burden would be present to test this dualpurpose graft. Due to a lack of osteoinductive agents, the defects in the VA/CPC group were not completely repaired, although no indication of infection was found in this group. By contrast, ossification and medullary cavity recanalization were observed in defects of the IC-VA/CPC group. Based on these results, we hypothesize that IC may be used as an effective osteoinductive agent for the treatment of fractures and bone defects.

In conclusion, these results demonstrate that IC-VA/CPC as a local antibiotic release system for treating infection-related bone disease in patients with open fractures displays several advantages. The CPC bone graft is injectable, provides a more sustained release of icariin or other osteoinductive molecules and is able to release antibiotics for more than 30 days. Thus, the dual-delivery approach may improve patient outcomes by providing osteoinductive agents and antibiotics until bone healing occurs. In addition, the slow, sustained release of antibiotics at the site of infection yields elevated local concentrations, while minimizing any risk of systemic toxicity. A variety of antibiotics may be selected to cover both Gram-negative and Gram-positive bacteria, thereby facilitating individualized chemotherapy. Furthermore, in contrast to expensive growth factors, icariin is low-cost. Finally, the biodegradability of the scaffold materials eliminates the requirement for a second surgical procedure for their removal.

\section{Acknowledgements}

This study was supported by the Key Projects of Science and Technology Pillar Program of Ningxia Province, China.

\section{References}

1. Bi L, Hu Y, Fan H, Meng G, Liu J, Li D and Lv R: Treatment of contaminated bone defects with clindamycin-reconstituted bone xenograft-composites. J Biomed Mater Res B Appl Biomater 82: 418-427, 2007.

2. Korkusuz F, Korkusuz P, Eksioglu F, Gursel I and Hasirci V: In vivo response to biodegradable controlled antibiotic release systems. J Biomed Mater Res 55: 217-228, 2001.

3. Shinto Y, Uchida A, Korkusuz F, Araki N and Ono K: Calcium hydroxyapatite ceramic used as a delivery system for antibiotics. J Bone Joint Surg Br 74: 600-604, 1992.

4. CornellCN,TyndallD, WallerS,Lane JMandBrauseBD:Treatment of experimental osteomyelitis with antibioticim-pregnated bone graft substitute. J Orthop Res 11: 619-626, 1993.

5. Moehring HD, Gravel C, Chapman MW and Olson SA: Comparison of antibiotic beads and intravenous antibiotics in open fractures. Clin Orthop Relat Res 372: 254-261, 2000. 
6. Guelcher SA, Brown KV, Li B, Guda T, Lee BH and Wenke JC: Dual-purpose bone grafts improve healing and reduce infection. J Orthop Trauma 25: 477-482, 2011.

7. Bi L, Cheng W, Fan H and Pei G: Reconstruction of goat tibial defects using an injectable tricalcium phosphate/chitosanin combination with autologous platelet-rich plasma. Biomaterials 31 : 3201-3211, 2010

8. Cui G, Li J, Lei W, Bi L, Tang P, Liang Y, Tao S and Wang Y: The mechanical and biological properties of an injectable calcium phosphate cement-fibrin glue composite for bone regeneration. J Biomed Mater Res B Appl Biomater 92: 377-385, 2010.

9. Urabe K, Naruse K, Hattori H, Hirano M, Uchida K, Onuma K, Park HJ and Itoman M: In vitro comparison of elution characteristics of vancomycin from calcium phosphate cement and polymethylmethacrylate. J Orthop Sci 14: 784-793, 2009.

10. Okada M, Sangadala S, Liu Y, Yoshida M, Reddy BV, Titus L and Boden SD: Development and optimization of a cell-based assay for the selection of synthetic compounds that potentiate bone morphogenetic protein-2 activity. Cell Biochem Funct 27: 526-534, 2009.

11. Fan JJ, Cao LG, Wu T, Wang DX, Jin D, Jiang S, Zhang ZY, Bi L and Pei GX: The dose-effect of icariin on the proliferation and osteogenic differentiation of human bone mesenchymal stem cells. Molecule 16: 10123-10133, 2011.

12. Zhao J, Ohba S, Komiyama Y, Shinkai M, Chung UI and Nagamune T: Icariin: a potential osteoinductive compound for bone tissue engineering. Tissue Eng Part A 16: 233-243, 2010.

13. Jiang F, Wang XL, Wang NL and Yao XS: Two new flavonol glycosides from Epimedium koreanum Nakai. J Asian Nat Prod Res 11: 401-409, 2009.

14. Nian H, Ma MH, Nian SS and Xu LL: Antiosteoporotic activity of icariin in ovariectomized rats. Phytomedicine 16: 320-326, 2009.
15. Zhao J, Ohba S, Shinkai M, Chung UI and Nangamue T: Icariin induces osteogenic differentiation in vitro in a BMP-and Runx2-dependent manner. Biochem Biophys Res Commun 369: 444-448, 2008

16. Fan J, Bi L, Wu T, Cao L, Wang D, Nan K, Chen J, Jin D, Jiang S and Pei G: A combined chitosan/nano-size hydroxyapatite system for the controlled release of icariin. J Mater Sci Mater Med 23: 399-407, 2012

17. Bitar M, Friederici V, Imgrund P, Brose C and Bruinink A In vitro bioactivity of micro metal injection moulded stainless steel with defined surface features. Eur Cell Mater 23: 333-347, 2012.

18. Li HB and Chen F: Separation and purification of epimedin A, B, $\mathrm{C}$, and icariin from the medicinal herb Epimedium brevicornum maxim by dual-mode HSCCC. J Chromatogr Sci 47: 337-340, 2009.

19. Larsson S and Bauer TW: Use of injectable calcium phosphate cement for fracture fixation: a review. Clin Orthop Relat Res 395: 23-32, 2002.

20. International Organization for Standardization: ISO 10993-12:2007: Biological evaluation of medical devices. Part 12: Sample preparation and reference materials. ISO, Geneva, Switzerland, 2007.

21. Yang L, Wang NL and Cai GP: Maohuoside A promotes osteogenesis of rat mesenchymal stem cells via BMP and MAPK signaling pathways. Mol Cell Biochem 358: 37-44, 2011.

22. Melicherčík P, Jahoda D, Nyč O, Klapková E, Barták V, Landor I, Pokorný D, Judl T and Sosna A: Bone grafts as vancomycin carriers in local therapy of resistant infections. Folia Microbiol (Praha) 57: 459-462, 2012.

23. Bert F, Leflon-Guibout V, Le GJ, Bourdon N and Nicolas MH: Emergence of vancomycin-dependent enterococci following glycopeptide therapy: case report and review. Pathol Biol (Paris) 57: 56-60, 2009 (In French). 